\title{
Improved Markov Chain MBER Detection for Steered Linear Dispersion Coded MIMO Systems
}

\author{
Shinya Sugiura ${ }^{\star}$, Nan $\mathrm{Wu}^{\dagger}$ and Lajos $\mathrm{Hanzo}^{\dagger}$ \\ ${ }^{\star}, \dagger$ School of ECS, University of Southampton, SO17 1BJ, UK, Tel: +44-23-8059-3125, Fax: +44-23-8059-4508 \\ Email: \{ss07r,nw04r,lh\}@ecs.soton.ac.uk, http://www-mobile.ecs.soton.ac.uk \\ *TOYOTA Central R\&D Labs., Inc., Aichi, 480-1192, Japan. Tel: +81-561-71-7163, Fax: +81-561-63-5258 \\ Email: sugiura@mosk.tytlabs.co.jp, http://www.tytlabs.co.jp/eindex.html
}

\begin{abstract}
In this paper, we present an iterative Markov Chain Minimum Bit Error Rate (MC-MBER) detection aided steered Linear Dispersion Coded (LDC) structure, which amalgamates three different Multiple-Input Multiple-Output (MIMO) functions, namely Space-Time Coding (STC), Spatial Division Multiplexing (SDM) and beamforming. Furthermore, the concept of using a novel a priori Log Likelihood Ratio (LLR) threshold based technique is invoked for the sake of further reducing the computational complexity imposed. Both the EXtrinsic Information Transfer (EXIT) chart analysis and our BER performance results demonstrate that the achievable performance is substantially improved upon increasing the number of iterations $I$. At BER = $1 \times 10^{-4}$, the required $E_{\mathrm{b}} / N_{0}$ value is about $2.4 \mathrm{~dB}$ apart from that of the maximum achievable rate in conjunction with $I=10$ in a rank-deficient system transmitting $Q=6$ QPSK modulated streams in $T=\mathbf{2}$ symbol durations with the aid of $N=\mathbf{2}$ receive antennas and $M=2$ transmit antenna arrays, where each of the arrays is composed of $L=2$ antenna elements. It is demonstrated that the computational complexity of the $a$ priori LLR threshold assisted MC-MBER detector is reduced by $38 \%$ at $\mathrm{BER}=1.4 \times 10^{-5}$ in comparison to that of the MC-MBER detector dispensing with the thresholding technique.
\end{abstract}

\section{INTRODUCTION}

When aiming for powerful and flexible wireless communication system design capable of achieving both a high spectrum efficiency as well as a high reliability, MultipleInput Multiple-Output (MIMO) systems employing an antenna array at the receiver and/or the transmitter have been widely investigated [1]. Such MIMO arrangements can be classified, according to their functions, into four categories, Space-Time Coding (STC), Spatial Division Multiplexing (SDM), Space Division Multiple Access (SDMA) and beamforming schemes.

Recent studies revealed that some combinations of the four MIMO techniques allow the system to simultaneously exploit several MIMO functions [2], [3]. Moreover, Hassibi et al. [4] developed a sophisticated MIMO space-time processing architecture referred to as Linear Dispersion Codes (LDC), which amalgamate the benefits of STC and SDM. The LDC has the capability of striking an attractive diversity-multiplexing tradeoff for an arbitrary number of antennas and constellation sizes [4], [5].

Several detection algorithms have been developed for coded and uncoded MIMO systems [1]-[9]. The most popular algorithm is the Minimum Mean Square Error (MMSE) scheme

The financial support of the EU under the auspices of the Optimix project and also that of the EPSRC is thankfully acknowledged.
[6], [7], which minimizes the MSE between the desired signal and the actual array output. In recent years, the Minimum Bit Error Rate (MBER) detector [8], [9] has also been investigated, which directly minimizes the BER, therefore outperforms the MMSE detector at the cost of a higher computational complexity.

Against this background, the novel contributions of this paper are as follows:

1) We present a reduced-complexity iterative Markov Chain based MBER (MC-MBER) detection aided generalized Multi-Functional Antenna Array (MFAA) structure, which amalgamates the three MIMO functions, namely STC, SDM and beamforming. More specifically, the MFAA is realized with the aid of LDC transmissions and beamforming aided reception.

2) The computational complexity of the MC-MBER detector is further reduced by introducing the novel concept of a priori Log Likelihood Ratio (LLR) thresholding, which facilitates an efficient implementation of the MC simulation [10].

3) The effects of the MC-MBER detector's parameters are investigated for the first time with the aid of EXtrinsic Information Transfer (EXIT) charts [11]. In addition, the maximum achievable rate of our system is evaluated based on our EXIT chart analysis, which is followed by the investigation of the achievable BER and the computational complexity imposed.

The remainder of the paper is organized as follows. The model of the proposed MFAA structure is presented in Section II. We discuss the improved MC-MBER design in Section III. In Section IV, we provide some simulation results. Finally, Section V concludes this paper.

\section{Signal MODEL}

Consider a MIMO system having $M$ transmit antennas and $N$ receive Antenna Arrays (AAs), where each of the receive AAs is composed of $L$ half-wavelength-spaced antenna elements. At the transmitter, a frame of source bits $b$ is first channel encoded and then interleaved by a random interleaver $\Pi$. The interleaved bits are Serial-to-Parallel (S/P) converted to $Q$ substreams and then mapped to QPSK symbols $\boldsymbol{s}(k)=$ $\left[s_{1}(k), \cdots, s_{Q}(k)\right]^{T}$. Next, the LDC-mapping block maps the input symbols $\boldsymbol{s}(k)$ to the space-time blocks $\boldsymbol{S}(k) \in \mathcal{C}^{M \times T}$ 


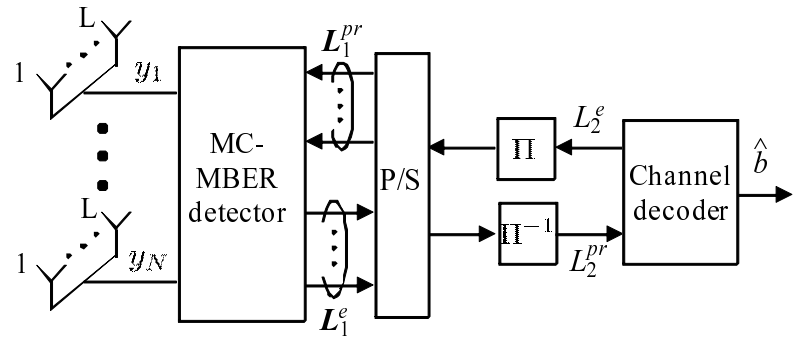

Fig. 1. Receiver structure of a steered linear dispersion coded uplink.

having the relation [4]

$$
\boldsymbol{S}(k)=\sum_{q=1}^{Q} \boldsymbol{A}_{q} s_{q}(k) .
$$

Each space-time block $\boldsymbol{S}(k)$ is transmitted over $T$ symbol durations by the $M$ transmit antennas. In this contribution, the LDC's dispersion matrices $\boldsymbol{A}_{q}$ are designed to maximize the Discrete-input Continuous-output Memoryless Channel (DCMC) capacity [12], given the parameters of the LDC $(M, N, T, Q)$.

Assuming frequency-flat block Rayleigh fading channels $\boldsymbol{H}(k) \in \mathcal{C}^{N L \times M}$ having independent channel realizations for each transmission block of $Q$ symbols, the received signal blocks $\boldsymbol{Y}(k) \in \mathcal{C}^{N L \times T}$ are expressed as

$$
\boldsymbol{Y}(k)=\boldsymbol{H}(k) \boldsymbol{S}(k)+\boldsymbol{N}(k),
$$

where each component of the Additive White Gaussian Noise (AWGN) blocks $\boldsymbol{N}(k) \in \mathcal{C}^{N L \times T}$ has a zero mean and a variance of $\sigma_{\mathrm{n}}^{2}$ per dimension. To elaborate a little further, the assumption of having a block fading channel requires selectively short transmitting blocks of $Q$ symbols and/or a low Doppler frequency. In this scenario, each transmission block experiences an AWGN channel associated with a different SNR.

Fig. 1 shows the receiver structure of our steered LDC aided MIMO system. Each of the $N$ AAs configures the beam pattern based on the Direction-Of-Arrival (DOA) information from the transmitter, resulting in a beamforming gain. The Channel Impulse Response (CIR) $h_{(n-1) L+l, m}(k)$ between the $m$ th transmit antenna and the $l$ th antenna element of the $n$th receive AA is given by $h_{(n-1) L+l, m}(k)=\alpha_{n, m}(k) \exp \{j \pi(l-$ 1) $\left.\sin \left(\phi_{m n}\right)\right\}$, where $\phi_{m n}$ is the average DOA of the $m n$th link and $\alpha_{n, m}(k)$ is a Rayleigh faded envelope. Assuming that the complex weight $w_{n, l}(k)$ corresponding to the $l$ th antenna element of the $n$th antenna array in the $k$ th LDC block is given by $h_{(n-1) L+l, m}^{*}(k)$, and that the weights $\boldsymbol{w}_{n}(k)=$ $\left[w_{n, 1}, \cdots, w_{n, L}\right]^{T}$ derived for each AA are normalized, the received signal blocks $\hat{\boldsymbol{Y}}(k) \in \mathcal{C}^{N \times T}$ at the AA are expressed as

$$
\hat{\boldsymbol{Y}}(k)=L \hat{\boldsymbol{H}}(k) \boldsymbol{S}(k)+\hat{\boldsymbol{N}}(k),
$$

where $\hat{\boldsymbol{H}}(k) \in \mathcal{C}^{N \times M}$ is composed of the entries $\alpha_{n, m}(k)$, and $\hat{\boldsymbol{N}}(k) \in \mathcal{C}^{N \times T}$ has a zero mean and a variance of $\sigma_{\mathrm{n}}^{2}$ per dimension. Here, (3) indicates a factor-of- $L$ processing gain, resulting from the beamforming at each receive AA.

According to [5], the block-based signal model (3) can be rewritten in a more tractable vectorial form as

$$
\boldsymbol{y}(k)=L \boldsymbol{H}^{\prime}(k) \boldsymbol{s}(k)+\boldsymbol{n}(k),
$$

where we have the relations

$$
\begin{aligned}
\boldsymbol{y}(k) & =\operatorname{vec}[\hat{\boldsymbol{Y}}(k)], \\
\boldsymbol{n}(k) & =\operatorname{vec}[\hat{\boldsymbol{N}}(k)], \\
\boldsymbol{H}^{\prime}(k) & =\tilde{\boldsymbol{H}}(k) \boldsymbol{\chi},
\end{aligned}
$$

with $\boldsymbol{\chi}=\left[\operatorname{vec}\left(\boldsymbol{A}_{1}\right), \cdots, \operatorname{vec}(\boldsymbol{A})_{Q}\right]$ and $\tilde{\boldsymbol{H}}(k)=\boldsymbol{I} \otimes \hat{\boldsymbol{H}}(k)$. The $\operatorname{vec}()$ operation denotes the vertical stacking of the columns of an arbitrary matrix and $\otimes$ denotes the Kronecker product.

The signals $\boldsymbol{y}(k)$ input to the MC-MBER detector are decoded using an iterative process, which exchanges soft information between two Soft-Input Soft-Output (SISO) decoders, namely the MC-MBER detector and the channel decoder of Fig. 1. The two decoders are separated by the S/P converter, the interleaver $\Pi$ and the deinterleaver $\Pi^{-1}$, as shown in Fig. 1. After a certain number of iterations $I$, the estimated bits $\hat{b}$ are output by the channel decoder.

\section{IMPROVED MARKOV CHAIN MBER DETECTOR}

In this section, we first review our detection algorithm, namely the MC-MBER detector, which combines the MBER detector [8], [9] and Markov chain simulation [10]. Then we introduce a novel concept, in which a priori information is utilized in the Markov chain simulation in order to reduce the associated computational complexity without substantially degrading its performance.

\section{A. MBER SISO Cancellation}

According to the soft interference cancellation principle [6], the estimated QPSK symbol $\hat{s}_{q}$ is calculated by

$$
\hat{s}_{q}=\boldsymbol{w}_{q}^{H}\left(\boldsymbol{y}-\boldsymbol{H}^{\prime} \overline{\boldsymbol{s}}+\bar{s}_{q} \boldsymbol{h}_{q}^{\prime}\right),
$$

where $\boldsymbol{w}_{q}$ represents the complex weights calculated by the MC-MBER detector and $\boldsymbol{h}_{q}^{\prime}$ represents the $q$ th column of $\boldsymbol{H}^{\prime}$. Furthermore, the signals $\bar{s}=\left[\bar{s}_{1}, \cdots, \bar{s}_{Q}\right]^{T}$ are computed from the a priori LLRs. In our MC-MBER detector, the weights $\boldsymbol{w}_{q}$ are obtained by minimizing the Bit Error Probability (BEP) function $P_{e}(\boldsymbol{w})$, which is expressed as

$$
\boldsymbol{w}_{q}=\arg \min _{\boldsymbol{w}} P_{e}(\boldsymbol{w})
$$

More specifically, as detailed in [8], (9) is efficiently minimized by means of the Simplified Conjugate Gradient (SCG) algorithm on the basis of the gradient of the BEP function, which is given by

$$
\begin{gathered}
\nabla P_{e}\left(\boldsymbol{w}_{q}\right)=\frac{1}{2 \sqrt{2 \pi} \sigma_{\mathrm{n}}} \sum_{m=1}^{N_{\mathrm{b}}} P\left(\boldsymbol{s}^{(m)}\right) \\
\cdot\left[\exp \left(-\frac{\left(\Re\left[\hat{s}_{q}^{(m)}\right]\right)^{2}}{2 \sigma_{\mathrm{n}}^{2}}\right) \operatorname{sgn}\left(\Re\left[s_{q}^{(m)}\right]\right)\left(\boldsymbol{w}_{q} \Re\left[\hat{s}_{q}^{(m)}\right]-\boldsymbol{y}^{\prime}\right)+\right.
\end{gathered}
$$




$$
\left.\exp \left(-\frac{\left(\Im\left[\hat{s}_{q}^{(m)}\right]\right)^{2}}{2 \sigma_{\mathrm{n}}^{2}}\right) \operatorname{sgn}\left(\Im\left[s_{q}^{(m)}\right]\right)\left(\boldsymbol{w}_{q} \Im\left[\hat{s}_{q}^{(m)}\right]+j \boldsymbol{y}^{\prime}\right)\right]
$$

with $\boldsymbol{y}^{\prime}=\boldsymbol{H}^{\prime} \boldsymbol{s}^{(m)}-\boldsymbol{H}^{\prime} \overline{\boldsymbol{s}}+\bar{s}_{q} \boldsymbol{h}_{q}^{\prime}$, where $P\left(\boldsymbol{s}^{(m)}\right)=$ $\Pi_{q} P\left(s_{q}=s_{q}^{(m)}\right)$ is the a priori probability of transmitting $\boldsymbol{s}^{(m)}$. Note that the norm of the detector weights $\boldsymbol{w}_{q}$ is normalized to unity in (10). In the full-complexity MBER detector, $N_{\mathrm{b}}=4^{Q}$ number of all the possible QPSK detection candidates $\boldsymbol{s}^{(m)}=\left[s_{1}^{(m)}, \cdots, s_{Q}^{(m)}\right]^{T}\left(m=1, \cdots, N_{\mathrm{b}}\right)$ are considered in (10), where the associated computational complexity increases exponentially upon increasing $Q$. By contrast, the MC-MBER detector employed in this paper efficiently approximates (10) by selecting a reduced amount of detection candidates $\boldsymbol{s}^{(m)}$ that most substantially contribute to the calculation of (10) with the aid of Markov chain simulation [10], as highlighted below.

\section{B. Markov Chain Representation}

As noted above, $N_{\mathrm{b}}$ number of the most likely detection candidates $\boldsymbol{s}^{(m)}\left(m=1, \cdots, N_{\mathrm{b}}\right)$ are inferred by means of Markov chain simulation in our steered LDC aided MIMO system. While several algorithms have been developed for the Markov chain representation [10], here we employ the most popular Gibbs-Sampler algorithm in this paper. A more detailed portrayal of the Gibbs-Sampler can be found in [10].

First of all, the QPSK signals $s[i=0]=$ $\left[s_{1}[0], \cdots, s_{Q}[0]\right]^{T}$, which represent the initial search pool of the Gibbs-Sampler, are generated from a randomly selected $2 Q$-bit binary sequence $\boldsymbol{b}[0]=\left[b_{1}[0], \cdots, b_{2 Q}[0]\right]^{T}$.

Then $N_{\mathrm{S}}$ number of detection candidates $\boldsymbol{s}[i] \quad(i=$ $\left.1, \cdots, N_{\mathrm{S}}\right)$ are calculated, where the $i$ th detection candidate $\boldsymbol{s}[i]$ is calculated in the $i$ th loop, conditioned on the $(i-1) \mathrm{st}$ detection candidate $s[i-1]$, on the a priori LLRs $\boldsymbol{L}_{1}^{\mathrm{pr}}=$ $\left[L_{1,1}^{\mathrm{pr}}, \cdots, L_{1,2 Q}^{\mathrm{pr}}\right]^{T}$ and on the received signal $\boldsymbol{y}$. To be more specific, the $q$ th element $b_{q}[i]$ of the binary signals $\boldsymbol{b}[i]$ in the $i$ th loop is calculated from the conditional probability

$$
P\left(b_{q}=+1 \mid \boldsymbol{b}_{-q}, \boldsymbol{y}, L_{1, q}^{\mathrm{pr}}\right)=\frac{1}{1+\alpha}
$$

with

$$
\alpha=\exp \left\{\frac{\left\|\boldsymbol{y}-\boldsymbol{H}^{\prime} \boldsymbol{s}_{1}\right\|^{2}-\left\|\boldsymbol{y}-\boldsymbol{H}^{\prime} \boldsymbol{s}_{2}\right\|^{2}}{2 \sigma_{\mathrm{n}}^{2}}-L_{1, q}^{\mathrm{pr}}\right\},
$$

where we have $\boldsymbol{b}_{-q}=\left[b_{1}[i], \cdots, b_{q-1}[i], b_{q+1}[i-\right.$ $\left.1], \cdots, b_{2 Q}[i-1]\right]^{T}$. Furthermore, the signals $s_{1}$ and $s_{2}$ are calculated from the binary sequences $b_{1}=$ $\left[b_{1}[i], \cdots, b_{q-1}[i], 0, b_{q+1}[i-1], \cdots, b_{2 Q}[i-1]\right]^{T}$ and $\boldsymbol{b}_{2}=$ $\left[b_{1}[i], \cdots, b_{q-1}[i], 1, b_{q+1}[i-1], \cdots, b_{2 Q}[i-1]\right]^{T}$, respectively. When a real-valued random variable $\xi$, which is uniformly distributed between 0 and 1 , happens to be lower than the probability $P\left(b_{q}=+1 \mid \boldsymbol{b}_{-q}, \boldsymbol{y}, L_{1, q}^{\mathrm{pr}}\right), b_{q}[i]$ is set to +1 , otherwise to -1 .

Following $N_{\mathrm{S}}$ iterations, the detection candidates generated during the last $N_{\mathrm{MC}}$ iterations are deemed to be the solution of the Gibbs-Sampler. Here, the first $N_{\mathrm{b}}=N_{\mathrm{S}}-N_{\mathrm{MC}}$ iterations represent so-called burn-in period, which allows the solution to converge to the most likely values. Additionally,

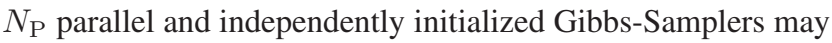
be implemented to avoid having a highly correlated detection candidate set when using a single Gibbs-Sampler. Note that introducing the parallel Gibbs-Sampler concept gives rise to an increase in the number of detection candidates from $N_{\mathrm{MC}}$ to $N_{\mathrm{P}} N_{\mathrm{MC}}$.

Finally, as the result of removing the overlapping detection candidates from the $N_{\mathrm{P}}$ parallel schemes, the most likely $N_{\mathrm{b}}$ number of detection candidates $\boldsymbol{s}^{(m)}$ are generated.

\section{A priori LLR Threshold Scheme}

Clearly, the above-mentioned Gibbs-Sampler is largely affected by the a priori information passed to it from the channel decoder, which indicates whether the corresponding bit is more likely to be +1 or -1 . For example, when the LLR $L_{1, q}^{\mathrm{pr}}$ is large positive value, $P\left(b_{q}=+1 \mid \boldsymbol{b}_{-q}, \boldsymbol{y}, L_{1, q}^{\mathrm{pr}}\right)$ in (11) is close to unity. Thus the bit $b_{q}[i]$ is set to 0 as a result of comparing $P\left(b_{q}=+1 \mid \boldsymbol{b}_{-q}, \boldsymbol{y}, L_{1, q}^{\mathrm{pr}}\right)$ to the random variable $\xi$.

Based on this fact, we introduce the novel concept of using an a priori LLR threshold based technique for the GibbsSampler, which reduces the complexity associated with the calculation of (11) by omitting the calculation of the lowprobability detection candidates. To be more specific, when the LLR $L_{1, q}^{\mathrm{pr}}$ is higher than a certain threshold value $\zeta>0$, the corresponding bit $b_{q}[i]$ is set to 0 without calculating (11). In the same way, if the LLR $L_{1, q}^{\mathrm{pr}}$ is less than the threshold value $-\zeta$, the bit $b_{q}[i]$ is set to 1 . It is plausible that a lower value of the threshold $\zeta$ leads to a lower complexity at the cost of a less accurate approximation of the Gibbs-Sampler. This trade-off is investigated with the aid of EXIT charts in the following section.

\section{Performance Analysis}

In this section, we present our performance results for the proposed MC-MBER detector aided steered LDC MIMO system employing $M=2$ transmit antennas, $N=2$ receive AAs, where each of the arrays has $L=2$ antenna elements. Each LDC block transmits $Q=6$ QPSK modulated symbols over $T=2$ symbol durations. The system employs a random interleaver having a length of 200,000 bits, and a half-rate Recursive Systematic Convolutional (RSC) channel code having a constraint length of 5 and the octally represented generator polynomials of $(35,23)$. Thus the total bandwidth efficiency is $3.0 \mathrm{bits} / \mathrm{symbol} / \mathrm{Hz}$. Note that the size of the equivalent channel matrix $\boldsymbol{H}^{\prime}$ is $(M T \times Q)=(4 \times 6)$ in this system, resulting in a challenging rank-deficient scenario.

\section{A. EXIT Chart Analysis}

Fig. 2 shows the EXIT curves of the MC-MBER detector at $\mathrm{E}_{\mathrm{b}} / \mathrm{N}_{0}=-5.8 \mathrm{~dB},-2.8 \mathrm{~dB}$ and $1.8 \mathrm{~dB}$ for the cases of $\left(N_{\mathrm{MC}}, N_{\mathrm{P}}, N_{\text {burn }}\right)=(10,1,5),(10,3,5)$ and $(10,5$, 5), where the a priori LLR thresholding technique is not activated. Furthermore, the EXIT curve of the MMSE detector is also portrayed. As shown in Fig. 2, all the three MC-MBER EXIT curves have the potential of providing a wider open 


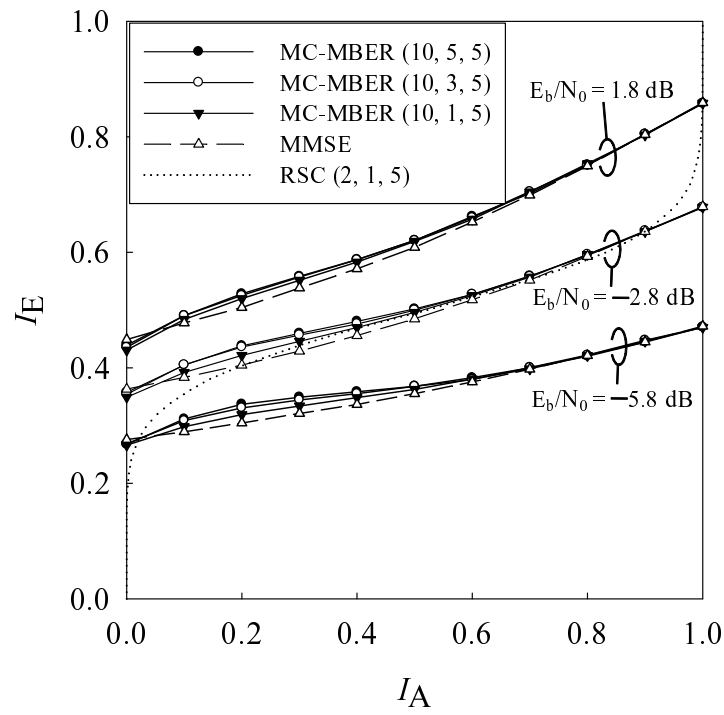

Fig. 2. EXIT curves of the MC-MBER and MMSE detectors in our linear dispersion coded MIMO system employing $M=2, N=2, L=2$, $T=2, Q=6$ and QPSK modulation. The system was operated at $\mathrm{E}_{\mathrm{b}} / \mathrm{N}_{0}=-5.8 \mathrm{~dB},-2.8 \mathrm{~dB}$ and $1.8 \mathrm{~dB}$. The parameters of the Gibbs-Sampler $\left(N_{\mathrm{MC}}, N_{\mathrm{P}}, N_{\text {burn }}\right)$ were set to $(10,5,5),(10,3,5)$ and $(10,1,5)$.

EXIT tunnel than that of the MMSE detector. Additionally, the MC-MBER detector exhibits a wider EXIT tunnel upon increasing the number of parallel Gibbs-Samplers $N_{\mathrm{P}}$. Since the difference between the curves of $\left(N_{\mathrm{MC}}, N_{\mathrm{P}}, N_{\mathrm{burn}}\right)=(10$, $3,5)$ and $(10,5,5)$ is small, the parameters $\left(N_{\mathrm{MC}}, N_{\mathrm{P}}, N_{\mathrm{burn}}\right)$ were set to $(10,3,5)$ in the rest of this paper.

Fig. 3 demonstrates our comparisons of the MC-MBER EXIT curves assisted by the a priori LLR thresholding technique employing the threshold values of $\zeta=0.1 \mathrm{~dB}, 1 \mathrm{~dB}$ and $2 \mathrm{~dB}$. The EXIT curve dispensing with the thresholding technique is also plotted for representing the upper limit. It can be seen that upon increasing the value $\zeta$, the EXIT curve is shifted upwards, approaching the 'no threshold' limit in the case of $\zeta=2 \mathrm{~dB}$.

\section{B. Maximum Achievable Rate}

It was shown in [13] that the maximum achievable rate is quantified by the area under the EXIT curve of the inner code. More specifically, the maximum achievable rate of our system is expressed as

$$
C\left(E_{\mathrm{b}} / N_{0}\right)=\frac{Q}{T} \log _{2}(\mathcal{M}) \cdot R\left(E_{\mathrm{b}} / N_{0}\right),
$$

where $\mathcal{M}$ is the constellation size employed and $R\left(E_{\mathrm{b}} / N_{0}\right)$ represents the area under the EXIT curve corresponding to a certain $E_{\mathrm{b}} / N_{0}$.

Fig. 4 shows the maximum achievable rate of the MCMBER detector used in our system, where the threshold $\zeta$ was set to $\zeta=2 \mathrm{~dB}$. The corresponding capacity curves of the Continuous-input Continuous-output Memoryless Channel (CCMC) [14] as well as the DCMC capacity [12] are also plotted in Fig. 4. While the CCMC capacity is a less restricted

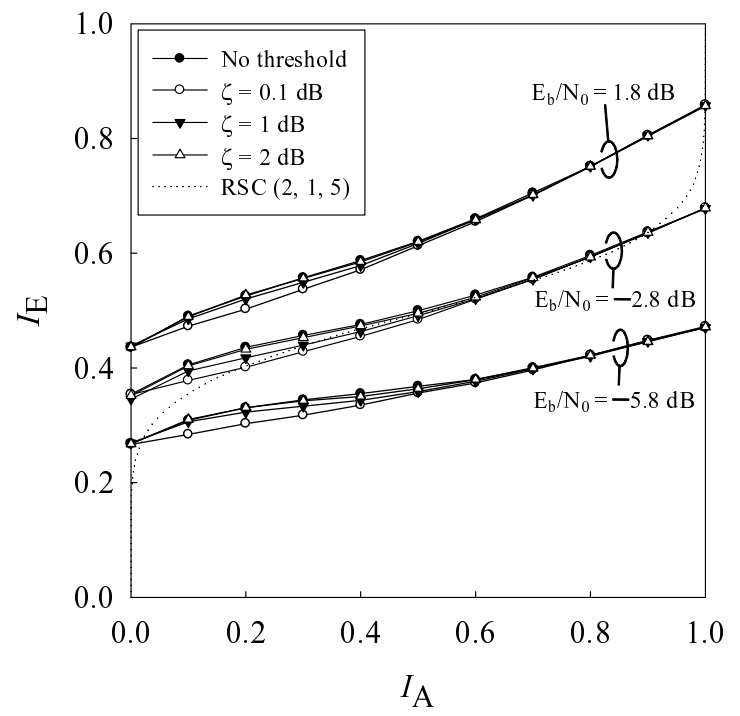

Fig. 3. EXIT curves of the a priori-LLR-threshold assisted MC-MBER detector in our steered LDC-aided MIMO system employing $M=2, N=2$, $L=2, T=2, Q=6$ and QPSK modulation. The system was operated at $\mathrm{E}_{\mathrm{b}} / \mathrm{N}_{0}=-5.8 \mathrm{~dB},-2.8 \mathrm{~dB}$ and $1.8 \mathrm{~dB}$. A priori LLR threshold $\zeta$ was set to $\zeta$ $=0.1 \mathrm{~dB}, 2 \mathrm{~dB}$ and $4 \mathrm{~dB}$, while keeping the parameters of the Gibbs-Sampler $\left(N_{\mathrm{MC}}, N_{\mathrm{P}}, N_{\text {burn }}\right)=(10,3,5)$.

bound, considering only the effect of the symbol power and the bandwidth, the DCMC capacity is a more practical bound, when designing channel-coded modulation schemes. Taking into consideration that the bandwidth efficiency of our system is $3.0 \mathrm{bits} / \mathrm{symbol} / \mathrm{Hz}$, the corresponding maximum achievable rate, the DCMC capacity and the CCMC capacity of our system were found to be attained at $E_{\mathrm{b}} / N_{0}=-3.0 \mathrm{~dB},-3.3$ $\mathrm{dB}$ and $-3.7 \mathrm{~dB}$, respectively.

\section{BER Performance and Computational Complexity}

Fig. 5 shows the achievable BER performance of the proposed MC-MBER detector having $\left(N_{\mathrm{MC}}, N_{\mathrm{P}}, N_{\text {burn }}\right)=(10$, $3,5)$ and $\zeta=2 \mathrm{~dB}$ in conjunction with $I=10$ iterations. The CCMC capacity and the maximum achievable rate are also plotted. It can be seen that the BER performance is substantially improved upon increasing the number of iterations $I$, where the $E_{\mathrm{b}} / N_{0}$ value required for achieving BER $=1 \times 10^{-4}$ is $2.4 \mathrm{~dB}$ away from the $E_{\mathrm{b}} / N_{0}$ of the maximum achievable rate in the case of $I=10$.

Fig. 6 shows the computational complexity required to calculate the detector weights $\boldsymbol{w}_{q}$ for the full-complexity MBER detector and for the MC-MBER detector both with and without the a priori LLR thresholding technique in our system, which was averaged over all the iterations $I$ at each SNR. The complexity was evaluated in terms of the number of realvalued operations, expressed as the sum of real-valued multiplications and real-valued additions. The MC-MBER detector exhibits more than 100 times lower complexity than that of the full-complexity MBER detector. Furthermore, at $E_{\mathrm{b}} / N_{0}=0.2$ $\mathrm{dB}$, where the corresponding BER is $1.4 \times 10^{-5}$, the a priori LLR threshold assisted MC-MBER detector achieves a further 


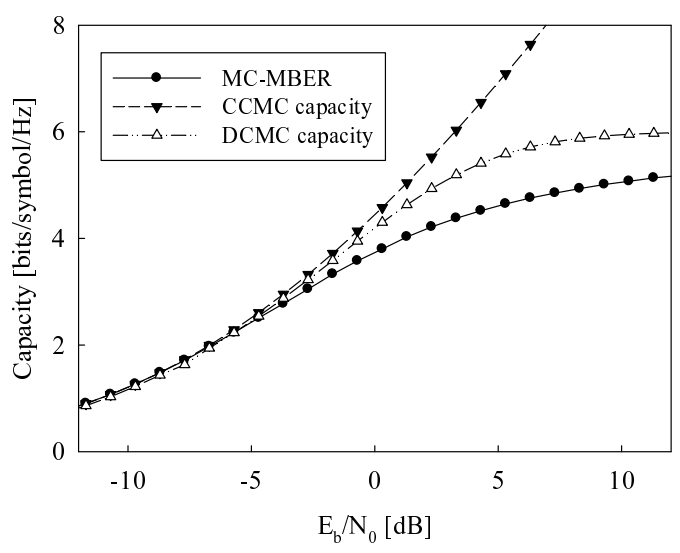

Fig. 4. Maximum achievable rates of the MC-MBER detector in our steered LDC-aided MIMO system employing $M=2, N=2, L=2, T=2$, $Q=6$ and QPSK modulation. The parameters of the MC-MBER detector were set to $\left(N_{\mathrm{MC}}, N_{\mathrm{P}}, N_{\mathrm{burn}}\right)=(10,3,5)$ and $\zeta=2 \mathrm{~dB}$. The corresponding CCMC and DCMC capacities are also plotted.

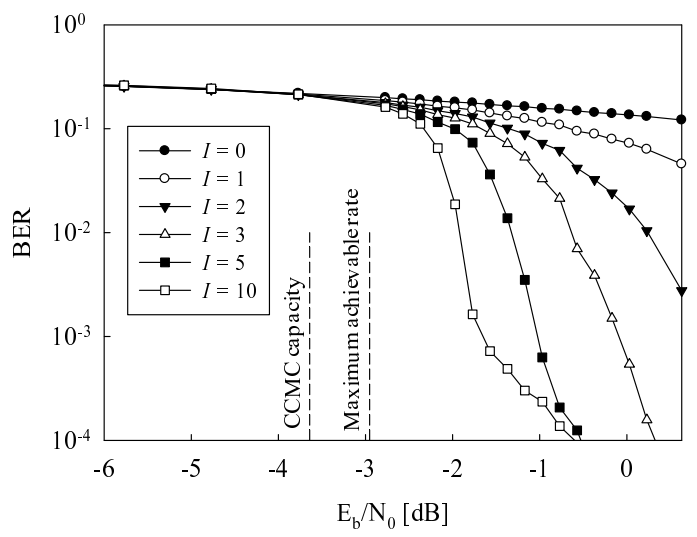

Fig. 5. BER performance of the MC-MBER detector having different number of iterations $I$ in our steered LDC-aided MIMO system employing $M=2$, $N=2, L=2, T=2, Q=6$ and QPSK modulation. The parameters of the MC-MBER detector were set to $\left(N_{\mathrm{MC}}, N_{\mathrm{P}}, N_{\mathrm{burn}}\right)=(10,3,5)$ and $\zeta$ $=2 \mathrm{~dB}$.

$38 \%$ reduced complexity in comparison to the MC-MBER detector which dispenses with the thresholding technique.

\section{CONCLUSION}

In this contribution, we proposed an iterative MC-MBER detected triple-functional MIMO system employing LDC transmission and beamforming reception. Additionally, the novel concept of the a priori LLR thresholding was invoked by the MC-MBER detector for the sake of reducing the computational complexity imposed. As a result of the EXIT chart analysis aided parameter optimization, the MC-MBER detector assisted by the a priori LLR thresholding technique achieved a $38 \%$ lower complexity at BER $=1.4 \times 10^{-5}$ in comparison to the detector dispensing with thresholding and more than 100 times lower complexity in comparison to the

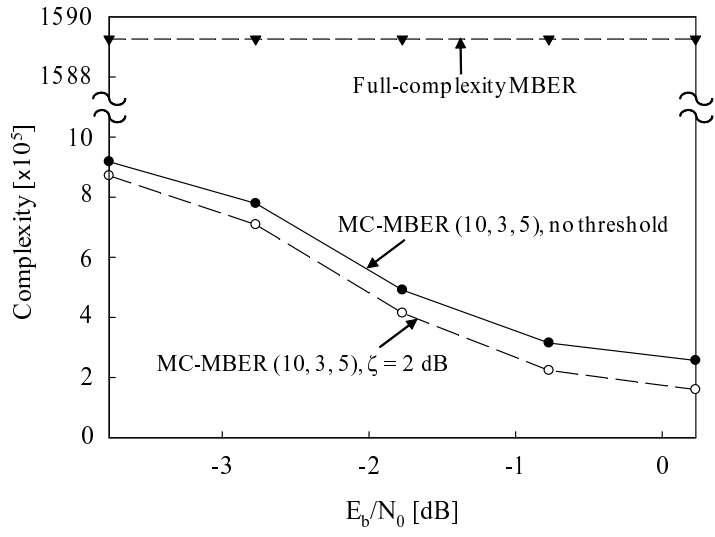

Fig. 6. Computational complexity expressed as the sum of real-valued additions and multiplications in our steered LDC-aided MIMO system employing $M=2, N=2, L=2, T=2, Q=6$ and QPSK modulation. The parameters of the MC-MBER detector were set to $\left(N_{\mathrm{MC}}, N_{\mathrm{P}}, N_{\mathrm{burn}}\right)=$ $(10,3,5)$ and $\zeta=2 \mathrm{~dB}$

full-complexity MBER detector in an $(M T \times Q)=(4 \times 6)$ element system having $M=2, N=2, L=2, T=2, Q=6$ and having QPSK modulation.

\section{REFERENCES}

[1] L. Hanzo, M. Münster, B. Choi, and T. Keller, OFDM and MC-CDMA for Broadband Multi-User Communications, WLANs and Broadcasting. John Wiley and IEEE Press, 2003.

[2] V. Tarokh, A. Naguib, N. Seshadri, and A. Calderbank, "Combined array processing and space-time coding," IEEE Trans. Inform. Theory, vol. 45, no. 4, pp. 1121-1128, May 1999.

[3] M. El-Hajjar, B. Hu, L.-L. Yang, and L. Hanzo, "Coherent and differential downlink space-time steering aided generalised multicarrier DSCDMA," IEEE Trans. Wireless Commun., vol. 6, no. 11, pp. 3857-3863, Nov. 2007.

[4] B. Hassibi and B. Hochwald, "High-rate codes that are linear in space and time," IEEE Trans. Inform. Theory, vol. 48, no. 7, pp. 1804-1824, July 2002.

[5] R. Heath Jr and A. Paulraj, "Linear dispersion codes for MIMO systems based on frame theory," IEEE Trans. Signal Process., vol. 50, no. 10 pp. 2429-2441, Oct. 2002

[6] X. Wang and H. Poor, "Iterative (turbo) soft interference cancellation and decoding for coded CDMA," IEEE Trans. Commun., vol. 47, no. 7, pp. 1046-1061, July 1999

[7] M. Tüchler, A. Singer, and R. Koetter, "Minimum mean squared error equalization using a priori information," IEEE Trans. Signal Process., vol. 50, no. 3, pp. 673-683, Mar. 2002.

[8] S. Chen, N. Ahmad, and L. Hanzo, "Adaptive minimum bit-error rate beamforming," IEEE Trans. Wireless Commun., vol. 4, no. 2, pp. 341348, Mar. 2005.

[9] S. Tan, S. Chen, and L. Hanzo, "On multi-user EXIT chart analysis aided turbo-detected MBER beamformer designs," IEEE Trans. Wireless Commun., vol. 7, no. 1, pp. 314-323, Jan. 2008.

[10] A. Doucet and X. Wang, "Monte Carlo methods for signal processing: a review in the statistical signal processing context," IEEE Signal Process. Mag., vol. 22, no. 6, pp. 152-170, Nov. 2005.

[11] S. ten Brink, "Convergence behavior of iteratively decoded parallel concatenated codes," IEEE Trans. Commun., vol. 49, no. 10, pp. 1727 1737, Oct. 2001.

[12] S. X. Ng and L. Hanzo, "On the MIMO channel capacity of multidimensional signal sets," IEEE Trans. on Vehicular Technology, vol. 55, no. 2, pp. 528-536, Mar. 2006.

[13] M. Tüchler, "Design of serially concatenated systems depending on the block length," IEEE Trans. Commun., vol. 52, no. 2, pp. 209-218, Feb. 2004.

[14] J. Proakis and M. Salehi, Digital communications. McGraw-Hill New York, 1995. 